Catherine Hassett, DO

Resident, Cleveland Clinic
Aron Gedansky, MD

Resident, Cleveland Clinic
MaryAnn Mays, MD

Center for Neurorestoration and Department of Neurology, Cleveland Clinic
Ken Uchino, MD

Cerebrovascular Center and Department of Neurology, Cleveland Clinic; Associate Professor, Cleveland Clinic Lerner College of Medicine of Case Western Reserve

University, Cleveland, $\mathrm{OH}$

\title{
Acute ischemic stroke and COVID-19
}

\section{Posted November 16, 2020}

\section{ABSTRACT}

Ischemic stroke may be a presenting feature of COVID-19. Its etiology remains unclear, but severe COVID-19 disease might increase the risk of large-artery strokes. More evidence is needed to substantiate the current reports and provide insights for optimal management.

\section{INTRODUCTION}

Although COVID-19 mostly causes lung injury, there are emerging data on related neurologic complications, including acute cerebrovascular disease.

\section{INCIDENCE AND RISK FACTORS}

A stroke incidence of $2.5 \%$ to $6 \%$ has been reported in retrospective studies of European and Chinese hospitalized COVID-19 patients. ${ }^{1-3}$

In a study of 219 patients hospitalized with COVID-19 in Wuhan, China, ${ }^{4}$ those with acute stroke $(\mathrm{N}=11 ; 5.0 \%)$ were more likely to be older, to present with severe symptoms, to have cardiovascular risk factors, such as hypertension and diabetes, and a history of cerebrovascular disease. In this report, fewer patients developed intracranial hemorrhage $(0.5 \%)$ than acute ischemic stroke $(4.6 \%)$.

Acute ischemic stroke was also reported during the earlier severe acute respiratory syndrome (SARS) and Middle East respiratory syndrome (MERS) epidemics., ${ }^{5,}$ Of 206 patients hospitalized for SARS in Singapore, 5 patients $(2.4 \%)$ had large-artery strokes. ${ }^{5}$ Three had no traditional cerebrovascular risk factors and 4 were critically ill, suggesting an association with the infection itself or its treatment. Potential causes of acute ischemic stroke in these patients included low ejection fraction from cardiac dysfunc-

The statements and opinions expressed in COVID-19 Curbside Consults are based on experience and the available literature as of the date posted. While we try to regularly update this content, any offered recommendations cannot be substituted for the clinical judgment of clinicians caring for individual patients.

doi:10.3949/ccjm.87a.ccc042 tion, disseminated intravascular coagulopathy, and marantic(nonbacterial thrombotic) endocarditis.

A recent case series from New York City supports this study's findings, as large-vessel ischemic strokes occurred in 5 patients with COVID-19 younger than 50 years. ${ }^{7}$ Each presented with acute stroke symptoms and lymphopenia and elevated inflammatory markers on admission laboratory tests, but 2 had no COVID. 19 symptoms.

\section{REVIEW OF PROPOSED ETIOLOGIES}

\section{Coagulopathy}

Patients with severe COVID-19 symptoms might be at risk of thromboembolic events from coagulopathy associated with COVID-19. In studies, patients hospitalized with COVID-19 have been reported to have increased coagulation activity marked by increased D-dimer concentrations. ${ }^{3,8-10}$ In one study, a D-dimer value greater than $1 \mu \mathrm{g} / \mathrm{mL}$ was associated with fatal outcome of COVID-19. ${ }^{9}$ In another study, patients with both cerebrovascular disease and SARS-CoV-2 had higher D-dimer levels than SARS-CoV-2 patients without cerebrovascular disease $(6.9 \mathrm{mg} / \mathrm{L}$ vs $0.5 \mathrm{mg} / \mathrm{L}, \mathrm{P}<.001){ }^{4}$

It remains unclear if elevated D-dimer values in patients with COVID-19 are directly associated with either arterial or venous ischemic stroke, or both. There is only 1 recent report of cerebral venous sinus thrombosis in a patient with severe COVID-19 who was successfully treated with anticoagulants. ${ }^{4}$

\section{Antiphospholipid antibodies}

Lupus anticoagulants and prolonged activated partial thromboplastin time are also frequently found in hospitalized patients with COVID-19, in whom the prevalence of lupus anticoagulant is $45 \%$ to $91 \% .^{9-12}$ There is no clear association between lupus anticoagulant and thrombosis in these studies, but a case series reported the finding of antiphospholipid antibodies in 3 critically ill COVID-19 patients with bilateral cerebral infarcts in multiple vascular territories. ${ }^{12}$ Laboratory investigations showed increased antiphos- 
pholipid antibodies, including anticardiolipin immunoglobulin A (IgA) and beta-2 glycoprotein IgM and $\mathrm{IgG}$, but without lupus anticoagulant.

This case series suggested that an acquired antiphospholipid syndrome was the underlying etiology, but unlike in the series of large-artery strokes in 5 young patients, ${ }^{7}$ these patients with antiphospholipid antibodies were over 60 years of age. ${ }^{12}$ The study did not provide information on other arterial or venous thromboembolism or report laboratory values for IgM or IgG antiphospholipid antibodies. In addition, 2 patients had prior strokes, but their underlying causes were not discussed. Finally, the questionable association of thrombosis with IgA antiphospholipid makes it difficult to determine a causal relationship.

These reports indicate the presence of antiphospholipid antibodies at variable frequencies, but likely higher than expected in the general population. Because their clinical significance is not yet known, these laboratory tests should not be routinely checked in COVID-19 patients without thrombosis.

\section{Vasculitis}

Other etiologies of ischemic stroke, such as virusinduced central nervous system vasculitis, were proposed when MERS and SARS patients had brain lesions in vascular patterns without a clear cerebrovascular cause. ${ }^{13,14}$ Postmortem histologic analysis of 3 patients with COVID-19 revealed lymphocytic endotheliitis within the endothelial cells of multiple organs, including the lungs, heart, kidneys, small intestine, and liver. ${ }^{15}$

The authors reported that endotheliitis may have caused microcirculatory vasoconstriction and endothelial dysfunction with consequent ischemia and apoptosis in the patients. ${ }^{15}$ Furthermore, direct viral infection of endothelial cells via angiotensinconverting enzyme 2 receptors, along with the host inflammatory response, may contribute to the wide spectrum of clinical sequalae of COVID-19.15 Histopathologic analysis of the central nervous system is needed to determine if SARS-CoV-2-related central nervous system vasculitis can occur from lymphocytic endotheliitis.

\section{MANAGEMENT}

Standard acute ischemic stroke management should be pursued, including reperfusion therapy with intravenous alteplase and mechanical thrombectomy.

Imaging findings in patients with COVID-19 with stroke have not revealed a single mechanism with evidence of small-vessel infarction, large-artery strokes, and cardioembolism. In addition, imaging options are likely limited in most patients, given the disease severity and limited access to magnetic resonance imaging (MRI).

One study reported unusual MRI findings in 13 patients with COVID-19 with encephalopathy. ${ }^{16}$ Imaging revealed leptomeningeal enhancement in 8 patients, and 3 patients were found to have silent cerebral ischemic strokes. Interestingly, all 11 patients who underwent perfusion imaging showed bilateral frontotemporal hypoperfusion. This study suggests that cerebral ischemia and meningoencephalitis might be underreported in patients with COVID-19 with encephalopathic presentations.

Close monitoring of characteristic laboratory findings of COVID-associated coagulopathy, such as elevated $\mathrm{D}$-dimer and fibrinogen, shortened prothrombin time and augmented partial thromboplastin time, and low international normalized ratio should be considered in suspected COVID-19 cases.

Appropriate diagnostic workup and antiplatelet agents for secondary stroke prevention should be considered.

Although prophylaxis of venous thromboembolism would be recommended for all nonambulatory hospitalized patients with COVID-19, including those with ischemic strokes, therapeutic anticoagulation for prevention of stroke remains uncertain. Current practices encourage an individualized decision based on imaging, timing, clinical context, and risk-benefit discussions.

Management strategies for anticoagulation in stroke patients with COVID-19 may change as more data are published.

\section{DISCLOSURES}

Dr. Hassett, Dr. Gedansky, and Dr. Uchino report nothing to disclose. Dr. Mays reported financial interests with Lilly (advisory), Teva, Amgen, Allergan (speaker's bureau), and DynamedPlus (consultant)

\section{REFERENCES}

1. Mao L, Wang M, Chen S, et al. Neurological manifestations of hospitalized patients with COVID-19 in Wuhan, China: a retrospective case series study. JAMA Neurology 2020 Apr 10; e201127. doi:10.1001/jamaneurol.2020.1127

2. Lodigiani C, Iapichino G, Carenzo L, et al. Venous and arterial thromboembolic complications in COVID-19 patients admitted to an academic hospital in Milan, Italy. Thromb Res 2020 Apr 23; 191:9-14. Online ahead of print. doi:10.1016/j.thromres.2020.04.024

3. Klok FA, Kruip MJ, van der Meer NJ, et al. Incidence of thrombotic complications in critically ill ICU patients with COVID-19. Thromb Res 2020; 191:145-147. doi:10.1016/j.thromres.2020.04.013

4. Wang D, Mao L, Jin H, Hu B. Acute cerebrovascular disease following COVID-19: a single center, retrospective, observational study. Stroke Vasc Neurol 2020; 5(3):279-284. doi: 10.1136/svn-2020-000431.

5. Umapathi T, Kor AC, Venketasubramanian N, et al. Large artery 
ischaemic stroke in severe acute respiratory syndrome (SARS). J Neurol 2004; 251(10):1227-1231. doi:10.1007/s00415-004-0519-8

6. Algahtani H, Subahi A, Shirah B. Neurological complications of Middle East respiratory syndrome coronavirus: a report of two cases and review of the literature. Case Rep Neurol Med 2016; 2016:3502683. doi:10.1155/2016/3502683

7. Oxley TJ, Mocco J, Majidi S, et al. Large vessel stroke as a presenting feature of COVID-19 in the young. N Engl J Med 2020; 382(20)e60. doi: 10.1056/NEJMc2009787

8. Guan WJ, NI Z, Yu Hu, W, et al. Clinical characteristics of coronavirus disease 2019 in China. N Engl J Med 2020; 382(18);1708-1729. doi:10.1056/NEJMoa2002032

9. Zhou F, Yu T, Du R. Clinical course and risk factors for mortality of adult inpatients with COVID-19 in Wuhan, China: a retrospective cohort study. Lancet 2020; 395(10229):1054-1062. doi:10.1016/ S0140-6736(20)30566-3

10. Bowles L, Platton S, Yartey N, et al. Lupus anticoagulant and abnormal coagulation tests in patients with Covid-19. N Engl J Med 2020; 383(3):288-290. doi:10.1056/NEJMc2013656

11. Harzallah I, Debliquis A, Drénou B. Lupus anticoagulant is frequent in patients with Covid-19. J Thromb Haemost 2020; 8(8):2064-2065. doi: $10.1111 /$ jth. 14867

12. Zhang $\mathbf{Y}$, Xiao $\mathbf{M}$, Zhang $\mathbf{S}$, et al. Coagulopathy and antiphospho lipid antibodies in patients with Covid-19. N Engl J Med 2020; 382(17):e38. doi:10.1056/NEJMc2007575

13. Arabi YM, Harthi A, Hussein J, et al. Severe neurologic syndrome associated with Middle East respiratory syndrome corona virus (MERS-CoV). Infection 2015; 43(4):495-501. doi:10.1007/ s15010-015-0720-y

14. Lau KK, Yu WC, Chu CC, et al. Possible central nervous system infection by SARS coronavirus. Emerg Infect Dis 2004; 10(2):342-344. doi:10.3201/eid1002.030638

15. Varga Z, Flammer AAJ, Steiger $\mathbf{P}$, et al. Endothelial cell infection and endotheliitis in COVID-19. Lancet 2020; 395(10234):1417-1418. doi:10.1016/S0140-6736(20)30937-5

16. Helms J, Kremer S, Merdji H, et al. Neurologic features in severe SARS-CoV-2 infection. N Engl J Med 2020; 382(23):2268-2270. doi: 10.1056/NEJMc2008597

Correspondence: Ken Uchino, MD, Cerebrovascular Center and Department of Neurology, Cleveland Clinic, 9500 Euclid Avenue, Cleveland, OH 44195; uchinok@ccf.org 